\title{
Searching for a hidden sector in multiparticle production at LHC
}

\author{
Miguel-Angel Sanchis-Lozano ${ }^{1, a}$, Edward Sarkisyan-Grinbaum²,3,b , Salvador Moreno-Picot ${ }^{4, c}$ \\ ${ }^{1}$ Departamento de Física Teórica, IFIC, CSIC-University of Valencia, 46100 Burjassot, Spain \\ ${ }^{2}$ Department of Physics, The University of Texas at Arlington, Arlington, TX 76019, USA \\ ${ }^{3}$ Department of Physics, CERN, 1211 Geneva 23, Switzerland \\ ${ }^{4}$ Departamento de Informática, ETSE, University of Valencia, 46100 Burjassot, Spain
}

\begin{abstract}
A hidden sector beyond the Standard Model can show up in multiparticle production altering inclusive correlations and factorial cumulants of multiplicity distributions. In this report such a study is advocated with a special emphasis on the searches at LHC.
\end{abstract}

\section{Introduction}

Most signatures of New Physics in colliders are expected to be found in hard events, on the transverse plane with respect to the beams' direction (i.e. emitting particles with high transverse momentum $p_{\perp}$ ), where background is much reduced. In this report, however, we focus on rather diffuse soft signals in $p p$ inelastic interactions, though expectedly tagged by hard decay products and appropriate cuts on events. For example, a non-standard state of matter from a Hidden Sector (HS) [1] might alter particle correlations [2-4] which can be measured to a large accuracy at the LHC.

Hadron interactions at high energy are usually considered as resulting from collisions of their constituent partons, likely dominated by pairwise parton interactions. In this work we study the effects of a new physics contribution on the conventional parton cascade. To this end, we extend the phenonemological approach of the Independent Pair Parton Interaction (IPPI) model [5, 6] including an extra step of a hypothetical new stage of matter associated with HS.

\subsection{Factorial moments of multiplicity correlations}

The study of inclusive particle correlations in multiparticle production can be performed by analyzing $n$-particle correlation functions and/or normalised factorial moments of multiplicity distributions [79]. Here we focus on the latter.

The normalized factorial moments of rank $q=2,3, \ldots$, are defined as

$$
F_{q}=\frac{\sum_{n} P(n) n(n-1) \cdots(n-q+1)}{\left(\sum_{n} P(n) n\right)^{2}},
$$

\footnotetext{
a e-mail: Miguel.Angel.Sanchis@ific.uv.es

be-mail: sedward@mail.cern.ch

ce-mail: Salvador.Moreno@uv.es
} 
Table 1. Probability distribution of the number of active pairs in proton-proton collisions for different $\mathrm{TeV}$ energies according to the IPPI model.

\begin{tabular}{cccccccc}
\hline$\sqrt{s}$ & $w_{1}$ & $w_{2}$ & $w_{3}$ & $w_{4}$ & $w_{5}$ & $w_{6}$ & $w_{7}$ \\
\hline $1.8 \mathrm{TeV}$ & 0.519 & 0.269 & 0.140 & 0.072 & 0.0 & 0.0 & 0.0 \\
$7.0 \mathrm{TeV}$ & 0.504 & 0.254 & 0.128 & 0.065 & 0.033 & 0.016 & 0.0 \\
$13 \mathrm{TeV}$ & 0.5020 & 0.2520 & 0.1265 & 0.0635 & 0.0319 & 0.0160 & 0.0080 \\
\hline
\end{tabular}

where $P(n)$ denotes the probability for $n$ final-state particles (charged hadrons).

The factorial moments represent any correlation between the emitted particles in events. To extract the genuine $q$-particle correlations, not reducible to the product of the lower-order correlations, one uses the normalised cumulant functions, or cumulants, defined as

$$
K_{q}=F_{q}-\sum_{r=1}^{q-1} \frac{(q-1) !}{r !(q-r-1) !} K_{q-r} F_{r} .
$$

Since $F_{q}$ and $\left|K_{q}\right|$ grow rapidly as the rank $q$ increases, it is convenient to consider the ratio

$$
H_{q}=\frac{K_{q}}{F_{q}},
$$

Factorial moments and cumulants have been extensively applied to the analysis of multihadron dynamics in different types of collisions, from $e^{+} e^{-}$to nucleus-nucleus interactions, in a broad range of energies [7-9]. In particular, normalized $H_{q}$ moments are extremely sensitive to the details of multiplicity distributions [10] and can be used to distinguish between different multiparticle production models and eventually the contribution of a HS as advocated in this paper.

\section{Multiparticle production as a multi-step cascade}

The IPPI model $[5,6]$ was proposed in order reproduce the moments of multiplicity distributions in $p p$ collisions at high energy with minimum adjustable parameters. The IPPI picture corresponds to a simplified 2-step scenario: parton binary collisions become seeds of independent cascades which hadronize (e.g. via string fragmentation) to the final-state multiparticle state.

Moreover, it is assumed that each pair parton interaction gives rise to a negative binomial distribution (NBD), while the total distribution is ultimately described by means of the weighted sum:

$$
P^{(2)}(n)=\sum_{j=1}^{j_{\max }} w_{j} \sum_{n_{i}} \prod_{i=1}^{j} P_{\mathrm{NBD}}\left(n_{i}, m^{(1)}, k^{(1)}\right)=\sum_{j=1}^{j_{\max }} w_{j} P_{\mathrm{NBD}}\left(n ; j m^{(1)}, j k^{(1)}\right),
$$

where $w_{j}$ denotes the probability for a $j$-pair interaction, $m^{(1)}$ and $k^{(1)}$ correspond to the mean multiplicity and dispersion for a single pair interaction, respectively. Note that no new adjustable parameters appear in Eq.(4) besides the distribution for $j$ binary parton interactions which can be evaluated if some model is adopted $[11,12]$.

In the IPPI, the probability for $j$ binary parton interactions per event is simply estimated as $w_{j}=$ $w_{1}^{j}$, where $w_{1}$ refers to a single pair, with the normalization condition $\sum_{j=1}^{j_{\max }} w_{j}=1$. In Table 1 we show the values of $w_{j}$ up to $w_{\max }=7$, corresponding to $p p$ collisions at the c.m. energy $\sqrt{s}$ of $13 \mathrm{TeV}$ taken from [6]. 


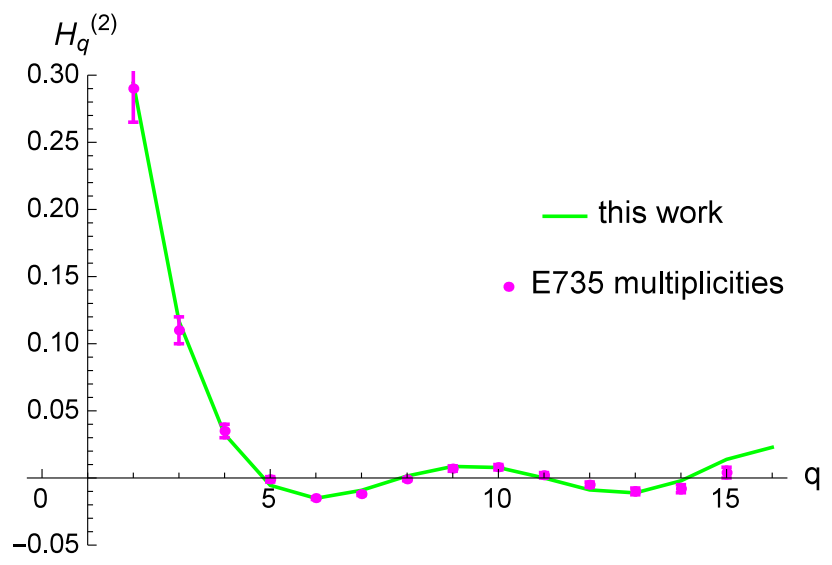

Figure 1. $H_{q}^{(2)}$ moments up to $q=16$ in $p \bar{p}$ collisions at $1.8 \mathrm{TeV}$ obtained in this work using expressions for a 2-step cascade and the same parameters as in $[5,6]$. Very good agreement is found with their results and experimental data [17], shown by circles with error bars.

\subsection{Two-step cascade}

Let us rewrite Eq.(4) for arbitrary particle production distributions and sources:

$$
P^{(2)}(n)=\sum_{N_{s}} P\left(N_{s}\right) \sum_{n_{i}} \prod_{i=1}^{N_{s}} P^{(1)}\left(n_{i}\right) .
$$

Here $n$ and $N_{s}$ denote the number of (charged) particles and sources, respectively. Note that the number of sources $N_{s}$ in Eq.(5) corresponds to the number of parton pair collisions $j$ in Eq.(4). In the notation used here, $P\left(N_{s}\right)$ stands now for the distribution of (fragmenting string) sources, equivalent to the parton pair interaction distribution $w_{j}$. Correspondingly, the average multiplicity can be written as $\langle n\rangle=\left\langle N_{s}\right\rangle m^{(1)}$ according to a 2-step description of multiparticle production.

On the other hand, the authors of $[5,6]$ benefit from a dramatic reduction of free parameters when assuming a weighted superposition of NBDs with shifted parameters, as can be seen in Eq.(4). In addition, since $m^{(1)}$ should be the same for any value of the rank $q$, only $k^{(1)}$ remains a free parameter ( $w_{\max }$ was determined using a particular model).

In order to make a comparison of the results of the current study and those from $[5,6]$, below we assume that all $P^{(1)}\left(n_{i}\right)$ are NBDs. Moreover, $P\left(N_{s}\right)$ and $w_{j}$ distributions can be formally identified.

The computation of high rank $F_{q}^{(2)}$ moments becomes extremely involved at large $q$. Therefore, we have written a Prolog code [13] which provides the expressions $F_{q}^{(p)}$ for any value of the rank $q$ and any number of steps $p$ in the cascade $F_{q}^{(p)}$, depending on the computer capacity available.

As shown below, we are able to reproduce (up to the percent level) the $H_{q}^{(2)}$ moments ${ }^{1}$ using the same values and assumptions as in Refs. [5,6]. This accordance suggests to proceed further in the approach given here by incorporating a new step in the parton cascade following the mIPPI scheme.

\footnotetext{
${ }^{1}$ The superindex $p$ in $H_{q}^{(p)}$ indicates the number of steps in the cascade: a two-step conventional cacade with $p=2$, and the three-step cascade with $p=3$ once a HS is included.
} 


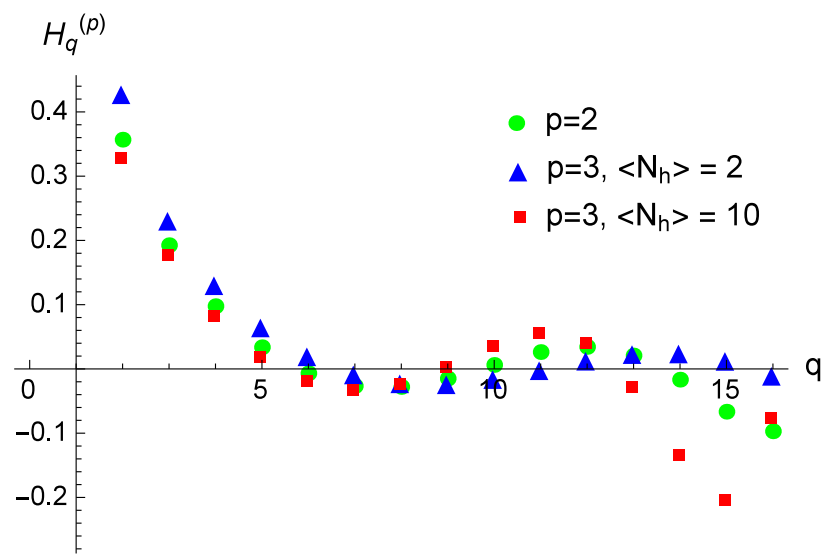

Figure 2. Predictions for $H_{q}^{(p)}$ moments as a function of the rank $q$ for $p p$ collisions at $\sqrt{s}=13 \mathrm{TeV}$. The circles correspond to a conventional 2-step cascade $(p=2)$ from extrapolation at lower energies using the IPPI model. The triangles and the squares correspond to a 3-step cascade $(p=3)$ using the mIPPI model (this work) with $\left\langle N_{h}\right\rangle=2$ and $\left\langle N_{h}\right\rangle=10$, respectively. A different pattern in the amplitude of the oscillations at high $q$ values can be clearly observed.

\subsection{Three-step cascade}

Let us now include an extra step in the cascade to simulate a hypothetical new stage of matter associated to a HS. The resulting multiplicity in a 3-step process should obey the following distribution:

$$
P^{(3)}(n)=\sum_{N_{s}} P\left(N_{s}\right) \sum_{n_{j}} \prod_{j=1}^{N_{s}} P^{(2)}\left(n_{j}\right) ; P^{(2)}(n)=\sum_{N_{h}} P\left(N_{h}\right) \sum_{n_{i}} \prod_{i=1}^{N_{h}} P^{(1)}\left(n_{i}\right),
$$

with $N_{h}$ denoting the number of active hidden sources in a collision. In what follows, for the sake of simplicity we assume that $P\left(N_{h}\right)$ follows a Poisson distribution, i.e. independent production of hidden sources resulting from binary parton interactions.

In other words, the probability distribution of parton interactions remains the same as in the conventional cascade (being already adjusted to reproduce experimental data in $p p$ collisions) while one adds another step subsequent to the initial binary parton interaction.

\section{$3 H_{q}$-moment oscillations as a function of the rank $q$}

QCD next-to-leading order calculations $[14,15]$ predict that the ratios $H_{q}$ defined in Eq.(3) oscillate as a function of the rank $q$, crossing the $q$-axis and becoming negative with $q_{\min } \approx 5$ at LHC energies, shifting to larger values at higher energies. This prediction has been tested against experimental data and found to be observed not only in $e^{+} e^{-}$collisions but also in a variety of colliding particles and energies, including $p p, p A$ and $A A$ collisions [16].

In Fig. 1 we plot the values of the $H_{q}^{(2)}$ moments $(q=2$ to 16$)$ for $\sqrt{s}=1.8 \mathrm{TeV}$ multiplicity data, obtained through Eqs.(2) and (3) from the expressions of $F_{q}^{(2)}$ (a 2-step cascade). We fix the parameters for the plot alike it is done in Ref. [5], i.e., assuming NBDs for all binary parton collisions with $k^{(1)}=4.4$, and $P\left(N_{s}\right)$ (equivalent to the $w$ distribution) from Table 1 . 


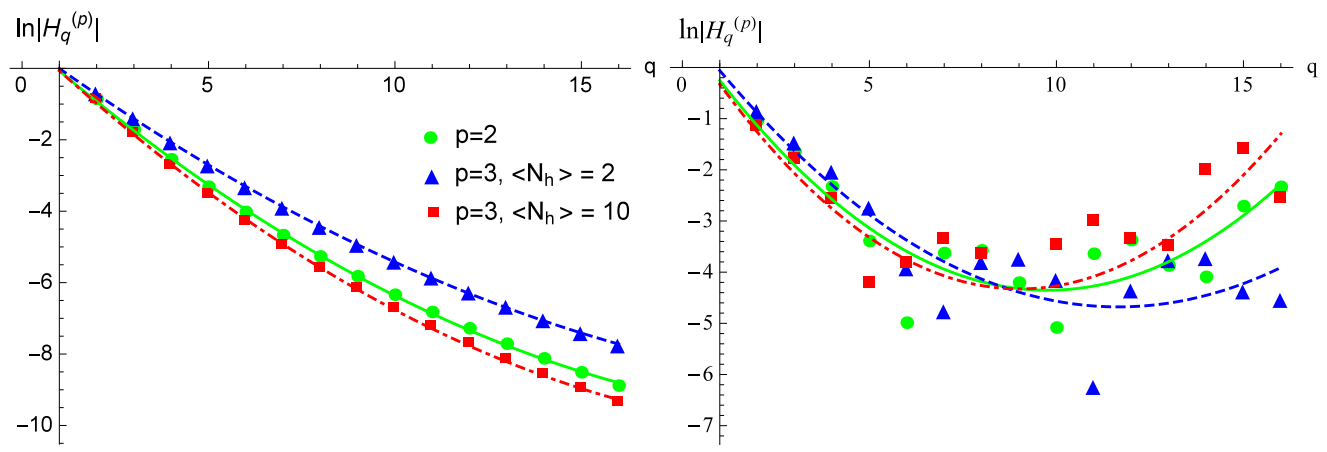

Figure 3. Values of $\ln \left|H_{q}^{(p)}\right|$ at $\sqrt{s}=13 \mathrm{TeV}$ versus $q$ for a 2-step scenario ( $p=2$, circles) and a 3-step scenario $(p=3)$ with $\left\langle N_{h}\right\rangle=2$ (triangles) and $\left\langle N_{h}\right\rangle=10$ (squares). Solid, dashed and dot-dashed lines correspond to parabolic fits, respectively. Left panel: superposition of NBDs with different reference values for their $k$ parameter (see [4]). Right panel: $P\left(N_{s}\right)$ incorporates the values of Table 1 (not the NBD case). Notice that there are no oscillations when all the distributions of the convolution are of the NBD type.

One can see the two minima in the Fig. 1. This oscillatory pattern is due to the fact that the probability distribution for the number of sources $P\left(N_{s}\right)$ (equivalently, the distribution for the number of parton pair collisions) does not follow a NBD. In case all the distributions are NBD, the resulting distribution turns out to be of the NBD type too and no oscillation pattern for $H_{q}^{(2)}$ shows up.

The overall good agreement with the results of Refs. [5, 6] and experimental data [17] suggests the further introduction of a new step in the cascade to be interpreted as a HS, thereby studying the eventual variation of the crossing points/minima and the amplitude of the $H_{q}$ oscillations.

\section{HS-cascade versus a conventional cascade}

In Fig. 2 three sets of points corresponding to different scenarios at $p p$ collisions at $\sqrt{s}=13 \mathrm{TeV}$ are shown. The circles correspond to a conventional cascade, while the triangles and squares correspond to an extra step in the mIPPI model setting $\left\langle N_{h}\right\rangle=2$ and $\left\langle N_{h}\right\rangle=10$, respectively. One can see that the crossing point (and minimum) moves by about one unit to the left for the $\left\langle N_{h}\right\rangle=10$, and by the same amount to the right for $\left\langle N_{h}\right\rangle=2$ compared to the case of a conventional cascade. Such an altered behaviour could become a hint of a HS affecting the parton evolution in multiparticle production, deserving a more detailed study.

Next we examine the amplitude of the $H_{q}$ oscillations as a function of the rank $q$, and its dependence on the parameters used in the mIPPI model as can be seen from in Fig. 2.

Depending on the number of hidden sources two different behaviours of the oscillation pattern of $H_{q}^{(3)}$ moments can be distinguished:

- For a small number of hidden sources, the oscillation amplitude becomes appreciably dumped for high $q$ values as compared to a conventional (2-step) cascade.

- For a large number of hidden sources, the oscillation amplitude is considerably larger for high $q$ values as compared to a conventional (2-step) cascade.

These conclusions are indeed confirmed in Fig. 3 where the values of $\ln \left|H_{q}\right|$ are plotted against $q$ for different scenarios depending on the type of the distributions used. The calculated points are 
shown together with the parabolic fits to them. One can see that the behaviour of the fitted curves is very different for different scenarios especially at large $q$ values.

The fitted curves pass through the points in the left panel, whereas the points scatter around the curves in the right panel. Hence no oscillations appear whenever all distributions in the superposition of Eqs.(6), including $P\left(N_{s}\right)$, are of the NBD type. This behaviour can be easily understood in the mIPPI model inasmuch the convolution of NBDs in Eq.(6) leads again to a NBD. Conversely, the oscillation pattern in the right panel emerges as a consequence of $P\left(N_{s}\right)$ not being a NBD.

\section{Summary}

Using the modified Independent Parton Pair Interaction we have studied the effect of a HS on the cumulant-to-factorial moment ratio $H_{q}$ of the multiplicity distributions of final-state particles in $p p$ collisions at the LHC. We conclude that a large (small) number of hidden sources would lead to an enhancement (softening) of the oscillation amplitude at high $q$ values. Moreover, the crossing of the $q$-axis and the minimum of the $H_{q}$-moments interpolating curve shifts to smaller (larger) $q$ values for a large (few) number of hidden sources.

\section{Acknowledgments}

Many thanks to the organizers of ISMD 2015 for a very pleasant working atmosphere and excellent organization. This work has been partially supported by MINECO under grants FPA2011-23596 and FPA2014-54459-P, and Generalitat Valenciana under grant PROMETEOII/2014/049.

\section{References}

[1] M. J. Strassler and K. M. Zurek, Phys. Lett. B 651, 374 (2007) [hep-ph/0604261].

[2] M.-A. Sanchis-Lozano, Int. J. Mod. Phys. A 244529 (2009) [arXiv:0812.2397 [hep-ph]].

[3] M.-A. Sanchis-Lozano and E. Sarkisyan-Grinbaum, arXiv:1409.5262 [hep-ph].

[4] M.-A. Sanchis-Lozano, E. K. Sarkisyan-Grinbaum, S. Moreno-Picot, arXiv:1510.08738 [hep-ph].

[5] I. M. Dremin and V. A. Nechitailo, Phys. Rev. D 70034005 (2004) [hep-ph/0402286].

[6] I. M. Dremin and V. A. Nechitailo, Phys. Rev. D 84034026 (2011) [arXiv:1106.4959 [hep-ph]].

[7] E. A. De Wolf, I. M. Dremin and W. Kittel, Phys. Rept. 2701 (1996) [hep-ph/9508325].

[8] I. M. Dremin and J. W. Gary, Phys. Rept. 349, 301 (2001) [hep-ph/0004215].

[9] W. Kittel, E. A. De Wolf, Soft Multihadron Dynamics (World Scientific, Singapore, 2005).

[10] R. Ugoccioni, A. Giovannini and S. Lupia, Phys. Lett. B 342387 (1995) [hep-ph/9410340].

[11] A. B. Kaidalov and K. A. Ter-Martirosyan, Phys. Lett. B 117247 (1982)

[12] S. G. Matinyan and W. D. Walker, Phys. Rev. D 59034022 (1999) [hep-ph/9801219].

[13] W.F. Clocksin and C.S. Mellish, Programming in Prolog, 5th edition. 2003. Springer-Verlag.

[14] I. M. Dremin, Phys. Lett. B 34195 (1994), [Phys. Lett. B 348711 (1995) (E)] [hep-ph/9408300].

[15] M. A. Buican, C. Forster and W. Ochs, Eur. Phys. J. C 31 (2003) 57 [hep-ph/0307234].

[16] I. M. Dremin, V. A. Nechitailo, M. Biyajima and N. Suzuki, Phys. Lett. B 403, 149 (1997) [hep-ph/9704318].

[17] E735 Collaboration, F. Turkot et al., Nucl. Phys A 525165 (1991) 